\title{
In vitro evaluation of the nematicidal value of Artocarpus altilis (Parkinson) var. seminifera and non seminifera and Terminalia cattapa L. against Haemonchus contortus
}

\author{
Carine Marie-Magdeleine ${ }^{\dagger}$, Maurice Mahieu, Marie-Laure Lastel and Harry Archimede \\ INRA UR143, 97170 Petit Bourg, France
}

\begin{abstract}
Introduction
Digestive parasities are the major pathologies of small ruminants in the Caribbean area. The frequent use of anthelmintics has led to strains of Haemonchus contortus resistant to benzimidazoles and avermectines throughout the tropics. Recent studies have shown that plants containing polyphenolics and particularly condensed tannins had anthelmintic properties. The current study is aimed at testing the antiparasitic potential of the leaf of three tropical trees containing phenolics and potentially exploitable in agroforestry systems: Artocarpus altilis (Parkinson) var. seminifera and var. non seminifera and Terminalia cattapa L., against the gastro intestinal nematode (GIN) Haemonchus contortus.
\end{abstract}

\begin{abstract}
Materials \& Methods
A phytochemical screening using classical methodology was performed on dead (D) and green (G) leaves. Quantitative determination of total polyphenols (TP) and condensed tannins (CT) were performed on D and $G$ leaves and on aqueous $(A)$ and methanolic (M) extracts of D leaf (Marie-Magdeleine, 2009). In vitro assays were performed according to Assis et al. (2003) methods. A and/or M extracts of D leaves were each tested on three different developmental stages of Haemonchus contortus using egg hatch assay (EHA), larval development assay (LDA), and adult worm motility assay (AWMA). The extracts were tested at three concentrations $(2.5,1.25$ and $0.625 \mathrm{mg} / \mathrm{mL})$ for EHA and LDA, and at four concentrations $(5,2.5,1.25$, and $0.625 \mathrm{mg} / \mathrm{mL})$ for AWMA. Each concentration repeated 5 times was compared with positive (albendazole for EHA and LDA, and levamisole for AWMA) and negative (phosphate buffer sample) controls. The data for in vitro assays were analyzed by the General Linear Model procedures with multiple comparisons (Bonferroni method) and regression, using the Minitab ${ }^{\circledR}$ Release 1.4 Software. P value $<0.05$ was taken for determination of significance level.
\end{abstract}

\section{Results}

Phytochemical screening analysis of leaves showed that $\mathrm{G}$ and $\mathrm{D}$ plant materials generally contained the same compounds, with a stronger revelation score for D samples. Artocarpus altilis (Parkinson) var. seminifera contained tannins, quinones, free anthracens, with a majority of proanthocyanidols, coumarins, phenols and triterpens and sterols. Artocarpus altilis (Parkinson) var. non seminifera contained phenols, coumarins, tannins, free anthracens, with a majority of flavans, proanthocyanidols, and triterpens and sterols. Terminalia cattapa L. contained free anthracens and a majority of tannins, phenols, flavans, proanthocyanidols, coumarins, and triterpens and sterols. In vitro assay showed that the A extract of Terminalia cattapa L. dead leaves exhibited egg hatching inhibition $(12.09 \% \pm 4.58)$ compared with the negative control $(P<0.001)$ and with a dose dependent effect $\left(P<0.001 ; R^{2}=53.0 \%\right)$. The A extract of $T$. cattapa L. dead leaf and the M extracts of T. cattapa L., $A$. altilis (Parkinson) var. seminifera and var. non seminifera D leaf exhibited larval development inhibition $(P<0.001)$ compared with negative control (respectively $24.62 \% \pm 6.86 ; 39.00 \% \pm 11.63 ; 21.01 \% \pm 4.66$ and $29.8 \% \pm 4.87$ inhibition). These four extracts of plant leaves exhibited dose-dependent effect within the concentration ranges tested (respectively $R^{2}=95.6 \% ; 97.3 \% ; 75.3 \%$ and $93.2 \%$; $P<0.001)$. The extracts all contained TP, respectively: 4.66; $4.92 ; 1.37$ and 0.40 (g/100 g of dry matter; gallic acid eq.). TC content was respectively: $2.78 ; 2.65 ; 3.96$ and 0.00 (g/100 g of dry matter; quebracho eq.). Quantitative chemical analysis results for TC and TP in whole G and $\mathrm{D}$ leaves are shown in Table 1.

Table 1 TP and CT content in G and D plant leaves

\begin{tabular}{|c|c|c|c|c|c|c|}
\hline \multirow[b]{2}{*}{ Chemical compound ( $\mathrm{g} / 100 \mathrm{~g}$ of dry matter) } & \multicolumn{2}{|c|}{ T. cattapa } & \multicolumn{2}{|c|}{ A. altilis var. seminifera } & \multicolumn{2}{|c|}{ A. altilis var. non seminiferc } \\
\hline & G & W & G & W & G & W \\
\hline TP (gallic acid eq.) & 3.50 & 3.33 & 0.76 & 0.39 & 0.74 & 0.58 \\
\hline CT (quebracho eq.) & 0.51 & 2.43 & 0.97 & 0.61 & 0.00 & 0.36 \\
\hline
\end{tabular}

\footnotetext{
${ }^{\dagger}$ E-mail: Carine.Marie-Magdeleine@antilles.inra.fr
} 


\title{
Conclusions
}

Phytochemical results indicate the predominance of the phenolic class compounds in the three vegetal resources tested. The anthelmintic effect observed in vitro in this experiment encourages conducting some studies in vivo with further studies on active compounds.

\section{Acknowledgments}

The authors gratefully acknowledge funding from Europe and Région Guadeloupe and Brigitte CALIF, Lucien PHILIBERT and Tatiana SILOU for technical assistance.

\section{References}

Assis LM, Bevilaqua CML, Morais SM, Vieira LS, Costa CTC and Souza JAL 2003. Veterinary Parasitology 117, 43-49.

Marie-Magdeleine et al. 2009. PhD Thesis.UAG Guadeloupe.

doi:10.1017/S2040470010000658

\section{Exploration and design of alternative feeding systems for livestock in the tropics by integrative system approaches}

\author{
J. C. J. Groot ${ }^{\dagger}$, H. von Keulen and S. J. Oosting \\ Wageningen University, The Netherlands
}

\section{Trends in cultivation of cereals and soy bean}

The use of cereals and soy meal, a by-product of oil production, as livestock feeds has increased tremendously during the last decades, and is expected to continue to grow. The increase in the supply of these feeds results from increases in both the productivity per area and the expansion of the cultivated area. Further increases in the productivity are possible, but in many situations in the tropics, constrained by lack of access to resources and technologies such as fertilizers, improved cultivars and water.

\section{Impact on the environment}

The negative impacts of these trends on the environment are related to the expansion of the cultivated area leading to destruction and fragmentation of ecosystems on fertile soils, and the cultivation in monocultures with high rates of fertilizer and pesticide application. Moreover, the intensive livestock production systems in which these feeds are used have severe additional detrimental effects to the environment, resulting from the application of pesticides, hormones and antibiotics, and the concentration of nutrients resulting in pollution of soils, water and air.

\section{Some possible alternatives}

In land-based ruminant production systems, a large potential exists for improved forage supply by restoration and sustainable management of grasslands, better management practices on crop lands, and the introduction of new plant types such as herbal and tree legumes. However, the realization of this potential is constrained by limitations in the resource endowment of many farmers. The production of meat from monogastric animals (poultry, pigs) is increasingly concentrated in large processing units and integrated into industrial supply chains. Such pork and poultry systems could benefit from responsible utilization of urban food wastes, agro-industrial by-products and non-conventional feed resources. In general, more sustainable development pathways could be reached when livestock production is reconnected to the land (and the people), either physically or through policies. Promising archetypes include mixed farming systems and urban agriculture.

\section{Criteria for alternative feeding systems}

Development of new feeding systems should originate from an integrated systems-oriented perspective on feed supply within the farm context, and the farm within the regional and higher scale settings. Moreover, long term concerns such as build-up of soil fertility should be explicitly included. Thus, multiple spatial and temporal scales should be addressed when developing new livestock production systems. More attention is needed for the sustainable use of locally and regionally available feed resources, and adaptation to and proper use of local circumstances and implementation of context specific practices. This could be combined with continuous system innovations, not only introducing new techniques, but also tuning existing and traditional practices.

\footnotetext{
${ }^{\dagger}$ E-mail: Jeroen.Groot@wur.nl
} 